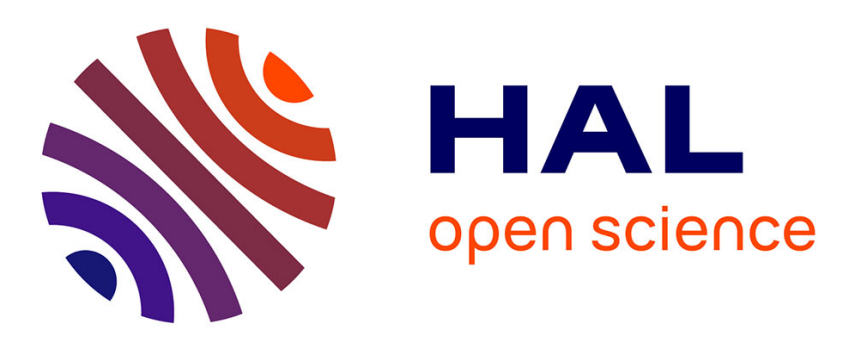

\title{
Entrer par le lexique dans la dimension dramatique du texte théâtral
}

\author{
Marie-Laure Elalouf
}

\section{To cite this version:}

Marie-Laure Elalouf. Entrer par le lexique dans la dimension dramatique du texte théâtral. Le Français Aujourd'hui, 2013. hal-03567044

\section{HAL Id: hal-03567044 https://hal.science/hal-03567044}

Submitted on 11 Feb 2022

HAL is a multi-disciplinary open access archive for the deposit and dissemination of scientific research documents, whether they are published or not. The documents may come from teaching and research institutions in France or abroad, or from public or private research centers.
L'archive ouverte pluridisciplinaire HAL, est destinée au dépôt et à la diffusion de documents scientifiques de niveau recherche, publiés ou non, émanant des établissements d'enseignement et de recherche français ou étrangers, des laboratoires publics ou privés. 


\section{Entrer par le lexique dans la dimension dramatique du texte théâtral}

Résumé : Lire une fable antique, réécrite à l'intention de lycéens, peut être une médiation pour la lecture de textes du théâtre classique et de leurs modèles gréco-latins, au programme de la classe de seconde. Mais comment faire appréhender les enjeux de la réécriture et la spécificité de chaque texte ? Autour du mot sacrifice, de ses emplois, constructions et des sentences où il s'inscrit, nous faisons le choix de trois entrées linguistiques mettant en lumière comment chacun actualise les potentialités de la fable d'Iphigénie.

Il peut sembler paradoxal de choisir Iphigénie pour «faire connaître les caractéristiques du genre théâtral et les effets propres au tragique »comme le préconisent les programmes de seconde ou pour aborder « le texte théâtral et sa représentation, du XVII à nos jours », second objet d'étude du programme de première. En effet, la pièce de Racine ne compte que de rares mises en scènes contemporaines : les manuels ne citent guère que celle de Yannis Kokos à la Comédie française en 1990 ; quant aux archives sonores, elles sont difficiles à obtenir. Mais les deux pièces d'Euripide, Iphigénie à Aulis et sa suite Iphigénie en Tauride, sont davantage représentées et une version contemporaine de la fable, Iphigénie ou le péché des dieux, a été écrite par Michel Azama à l'intention de lycéens. C'est l'existence de ces trois versions, trace de la «persistance de la mémoire » qui a déterminé le choix de proposer à des enseignants en formation la réflexion que cet article développe'. Nous montrerons dans un premier temps comment ce choix a permis d'articuler, pour de futurs enseignants de français, formation littéraire, linguistique et didactique. Puis nous décrirons les axes d'investigation que nous avons suivis.

\section{De l'université à la classe de français : des constantes}

Lorsque l'on aborde avec des étudiants avancés l'objet d'étude de la classe de seconde « La tragédie et la comédie au XVII siècle : le classicisme », on constate qu'ils ont surtout des œuvres classiques une connaissance sous forme d'extraits, dont ils ont mené l'étude analytique au lycée ou à l'université. Confrontés à d'autres extraits dans des manuels actuels, ils peinent à en justifier la pertinence et à établir des liens avec des oeuvres d'autres époques. Sont majoritairement convoqués pour la lecture analytique les relevés de champs lexicaux : quel que soit le genre du texte, ces listes sont souvent le point de départ ou un passage obligé du commentaire, avec les figures et les marques d'énonciation.

Les pratiques des étudiants en formation reflètent en cela des constantes observées dans les manuels de lycée, et que l'examen des manuels de 2011 conforte. Le lexique est abordé de façon rapide dans les pages consacrées à l'étude de la langue : les relations de forme et de sens entre les mots sont présentées sous forme de définitions suivies d'exemples, reprises au programme de collège. Les chapitres sur les spécificités du texte théâtral abordent la double énonciation, énumèrent une liste de termes pour désigner les répliques, mais n'évoquent jamais les négociations de sens qui se jouent entre les personnages et avec le public, pas plus

\footnotetext{
' Le travail présenté ici est une partie d'une élaboration plus complète proposée par Solange Bornaz, Sylviane Ahr et Denise Schröpfer et moi-même dans le cadre d'une journée d'étude sous le titre : Iphigénie (Euripide, Racine et Azama) : approches linguistiques, didactiques et mise en voix. Seule la contribution de Sylviane Ahr a été publiée en ligne : http://www.versailles.iufm.fr/pdf/publi/documents/iphigenie_sylviane.pdf. L'existence d'une riche iconographie permet des prolongements en histoire des arts.
} 
que les conflits sémantiques qui peuvent éclater de manière spectaculaire au théâtre alors que la polyphonie est moins facilement perceptible dans d'autres genres de discours.

En lecture analytique, l'entrée lexicale se fait sur le mode du relevé. Il s'agit tantôt de justifier l'appartenance à un registre («Relevez les termes relevant du registre pathétique », Labouret, 2011), tantôt d'orienter vers une interprétation psychologisante («Relevez le lexique de l'argent. Montrez que l'argent est une obsession pour les personnages », Abens, 2011) ou symbolique ( «Relevez dans ce texte le lexique ayant trait à la nature. En quoi est-elle synonyme de liberté », op. cit.). Le terme de champ lexical est encore employé (Daumas, Sivan, 2011) mais il cède le pas devant celui de lexique, ce qui résulte sans doute d'une lecture hâtive des programmes qui indiquent: "on fait acquérir aux élèves un lexique favorisant l'expression d'une pensée abstraite ». Il s'agit pour les élèves de s'approprier par des opérations de décontextualisation et de recontextualisation et inversement, un ensemble organisé d'unités lexicales, disponibles pour l'expression de la pensée abstraite, non des listes de vocabulaire : c'est le passage d'un emploi plus ou moins monosémique selon les genres discours (ex. «nature » dans la consigne précédente ) à une polysémie en langue (le champ sémantique de «nature ») et à un réemploi dans d'autres discours qui enrichit le concept tout en affinant les nuances d'emploi.

Or en tant que polylogue, le texte dramatique est une remarquable mise en scène des tensions entre différentes acceptions d'un même vocable, et plus encore le texte de tragédie qui repose sur l'affrontement de deux systèmes de références et de valeurs (Louvat, 1997). Son étude peut être un levier pour l'acquisition du lexique abstrait comme pour l'interprétation des textes. Certaines consignes relevées dans des manuels récents vont dans ce sens. Parfois c'est à l'élève de trouver l'entrée lexicale. À propos de la Médée d'Euripide, il est demandé pourquoi on peut parler de scène d'affrontement et c'est à lui de saisir la tension entre deux conceptions du bonheur :

«MÉDÉE

Et pour moi ? Une vie heureuse qui m'afflige et une prospérité qui me déchire le cœur ! JASON

(...) Arrive à ne plus considérer comme affligeante des situations favorables et à ne plus te croire malheureuse quand ton sort est heureux. » (Sivan, 2011).

Parfois, l'attention de l'élève est plus directement attirée vers un choix de désignation: «Comment le meurtre de Lorenzo est-il désigné dans cet extrait ? (Abens, 2011) et l'enjeu interprétatif précisé «Comment la mort des deux enfants est-elle désignée par les différents personnages ? En quoi le visage de la révolution dépend-il de cette désignation ? » (op. cit. à propos des Justes de Camus).

Par ailleurs, le texte dramatique, en tant que réécriture, permet d'observer l'évolution du lexique, autre perspective d'étude esquissée dans les programmes 2011 mais très peu développée dans les manuels. Les programmes précédents faisaient une place plus importante au développement de la conscience sémantique en langue (« habituer les élèves à raisonner de cette manière, non référentielle, sur le sens des mots constitue un des moyens de la prise de conscience sémantique ») et en discours ( «l'usage et l'histoire qui façonnent les assemblages et les significations »). Mais cette perspective a été peu travaillée, se limitant à quelques remarques dans les séries littéraires, alors même que le développement de cette conscience sémantique participe du développement d'une culture littéraire et d'une conscience esthétique, finalités des programmes. 
Le travail présenté ici poursuit trois objectifs : faire lire à des étudiants plusieurs réécritures d'une fable antique dans leur intégralité et observer comment le mot sacrifice s'y actualise, traversé dans chaque pièce de tensions et se chargeant de réécriture en réécriture de nouvelles interprétations. Parallèlement, la lecture des trois pièces a été proposée à des lycéens (Ahr, 2008) et une réflexion a été menée avec les étudiants sur le choix des extraits et les modalités de travail possible au lycée (lecture tabulaire et comparaisons, théâtre image). On fait l'hypothèse que des étudiants doivent avoir expérimenté certaines pratiques d'observation de la langue pour en mesurer les enjeux en termes d'apprentissage et qu'en leur proposant des tâches ajustées à leur propre maturité intellectuelle, on suscite une réflexion exigeante sur les adaptations didactiques possibles avec des lycéens.

\section{Trois variantes d'une même histoire}

La lecture croisée des œuvres a donc été proposée conjointement à des étudiants et des élèves de lycée. Ceux-ci ont été sensibles au texte contemporain qui, selon leurs propos, «parle de la jeunesse et s'adresse à la jeunesse » et sont progressivement entrés dans les textes plus anciens. Une même fable mais trois « acheminements » et trois dénouements différents. Chez Euripide, la substitution merveilleuse d'une biche à la victime laisse attendre une suite, Iphigénie en Tauride : qu'advient-il d'Iphigénie, une fois devenue «la capture d'un dieu » (v. 1615) ? On sait que Racine a refusé cette «machine » au nom de la vraisemblance. Refusant de « souill[er] la scène par le meurtre horrible d'une personne aussi vertueuse et aussi aimable qu'il fallait représenter Iphigénie » (préface de 1675), il a préféré inventer un nouveau personnage, Ériphile, greffant sur l'action principale, transmise par la tradition, une action secondaire : la recherche de ses origines par cette jeune captive et sa rivalité amoureuse avec Iphigénie. Les contemporains de Racine ont été sévères à l'égard de cette violation des lois de la réécriture. Le suicide d'Ériphile, découvrant que l'oracle s'appliquait à elle au moment même où Iphigénie devait périr, contrevient au principe que Pierre Corneille, dans son Discours de la tragédie (1660) reprend au chapitre 14 de la Poétique d'Aristote, affirmant «qu'il ne faut point changer les sujets reçus et que Clytemnestre ne doit point être tuée par un autre qu'Oreste ». Dans la réécriture de Michel Azama, le couteau de Kalkhas² n'épargne pas Iphigénie qui crie sa révolte jusqu'au dernier souffle :

« Comme il serait beau le sang du bourreau

si l'homme se risquait à la révolte».

Comment faire appréhender à des élèves de lycée les enjeux de la réécriture d'une même fable ? Sans doute faut-il revenir sur le sens de la réécriture à l'âge classique avant de déterminer des modalités didactiques. Si l'imitation est la règle dans l'esthétique classique, elle appelle la variante, comme le montre Pierre Bénichou (1967) : "Considérons donc les chefs d'oeuvre [du théâtre classique] comme des remaniements autant que comme des créations. Ces histoires dès longtemps consacrées, où vivent des personnages d'avance connus, sont comme des sujets d'exercice que le public propose incessamment aux auteurs en leur demandant de les repenser à l'usage du présent. Ce que chaque version apporte n'a son plein sens que par rapport aux autres ». Et Marc Escola (1998 : 21) de conclure : «On ne lira pas exactement l'Iphigénie d'Euripide comme la source de l'Iphigénie de Racine : il s'agira plutôt de voir ce que Racine et Euripide retiennent du sujet d'Iphigénie, de quelles possibilités dramatiques celui-ci se trouve potentiellement riche, quels ont été les possibles effectivement exploités par l'un et/ou l'autre des deux dramaturges, lesquels ont été refusés. Une telle confrontation pousse en effet à examiner aussi bien les solutions retenues que celles qui ont été écartées, et la scène refusée s'avère parfois plus importante pour l'intelligence d'une

\footnotetext{
${ }^{2}$ Graphie choisie par l'auteur, différente retenue par Racine : « Calchas ».
} 
structure dramatique que celle qui a été finalement adoptée. En d'autres termes, Euripide et Racine se trouvent avoir écrit deux tragédies parmi les Iphigénies possibles. On verra qu'il en est bien d'autres. »

Si l'on se reporte à l'introduction, écrite par Michel Azama en manière de préface, la volonté de repenser la fable antique à l'usage du présent est manifeste : «Avec Iphigénie, cette armée qui attend de partir à la guerre, c'est une fois de plus toutes les épées de Damoclès suspendues au-dessus de la tête de l'humanité. Que ce vieux mythe soit toujours neuf, quelle lapalissade! » Pour permettre aux élèves d'appréhender chacun des textes comme un développement possible de la signification de la fable, dans un contexte historique et culturel donné, nous avons choisi une entrée lexicale : le mot polysémique sacrifice, indissociable du sujet d'Iphigénie et du dénouement de la tragédie. C'est à une lecture tabulaire des trois pièces qu'ils sont invités, pour découvrir comment chacune actualise les potentialités de la fable d'Iphigénie. Nous partirons d'une approche sémantico-référentielle : que désigne le mot sacrifice dans chacun des textes et comment est-il déterminé ? Puis nous reviendrons à chacun des contextes : le sacrifice prend-il sens par rapport à un autre ? Le mot ayant des acceptions différentes selon les actants qu'il met en jeu, nous nous interrogerons sur les constructions du verbe sacrifier. Enfin, nous reviendrons à l'écriture dramatique en cherchant comment, à travers des sentences, s'exprime la valeur du sacrifice.

\section{Des sens de sacrifice aux sens du sacrifice}

\section{Sacrifice, un mot construit}

Une recherche dans le Robert Brio (2004), qui propose une analyse comparative des mots, permet de dégager deux éléments de composition issus du latin :

SACRI- Élément qui signifie « sacré ». Il apparaît dans les mots : sacrifier (sacrifié), sacrifice (sacrificateur), sacrilège.

(I)FI(C)- Élément qui signifie «faire», syn fac(t), fect(u), -urg. Il apparaît dans les verbes à base nominale dans la finale -fier (cocufier, liquéfier, modifier, pacifier, personnifier), dans des noms (amplificateur, bénéfice, codifcation...) et dans des adjectifs (artificiel, déficient, honorifique, magnifique...)

\section{SACRIFICE : SACRI- ; (I)FI(C)}

1. Offrande rituelle à la divinité, caractérisée par la destruction (réelle ou symbolique) ou l'abandon volontaire de la chose offerte. Faire des sacrifices aux dieux. Sacrifices d'animaux. Sacrifices humains. $\rightarrow$ immolation. Victime brûlée, offerte en sacrifice. (Robert Brio, 2004)

Une recherche dans un dictionnaire encyclopédique spécialisé, le Dictionnaire des mythologies (sous la direction d'Yves Bonnefoy, 1981) permet de revenir au sens originel du rituel :

«Sacrum facere : une part est volontairement soustraite au monde profane pour être offerte à la divinité. À quelle fin ? Sans doute, selon la mentalité des origines, afin de conforter les dieux qui, dans la conception romaine, sont solidaires des hommes. »

\section{Perpétuation/ détournement du rite}


Dans chacun des textes sont présents le sacrificateur et la victime, l'instrument et le lieu du sacrifice, le couteau et l'autel.

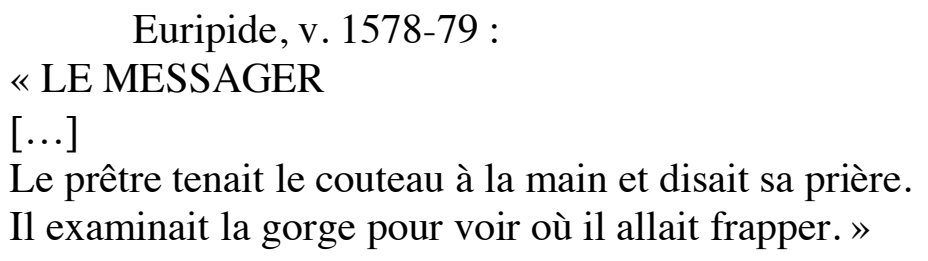

Azama, Dernières paroles, p. 65

«Le vent monte jusqu'à la tempête.

Iphigénie est sur l'autel.

Kalkas tient le couteau sacrificiel.»

\section{«ULYSSE}

Racine, V-6, 1775-76 :

$[\ldots]$

Arrête a-t-elle dit et ne m'approche pas.

Le sang de ces héros dont tu me fais descendre

Sans tes profanes mains saura bien se répandre.

Furieuse, elle vole, et sur l'autel prochain,

Prend le sacré couteau, le plonge dans son sein. »

Mais le texte de Racine tranche en ceci que la victime s'immole elle-même en déniant au prêtre toute légitimité. Elle devient ainsi impropre au sacrifice qui exige que la victime soit irréprochable pour convenir à la divinité, vierge ou animal sans défaut physique. André Green (1967 : 170) le souligne avec force : «il est clair, pour quiconque s'arrête à la signification d'un sacrifice, que la mort d'Ériphile ne peut en tenir lieu. D'abord parce qu'elle est un objet impropre à un tel rituel. Les exigences requises pour une telle cérémonie stipulent toujours que la victime doit être dépourvue de tout défaut ou de toute imperfection. C'est l'innocence qu'on sacrifie et ce qui doit périr doit être exempt de toute marque que déjà la Nature aurait imprimée sur un être, pour lui signifier une disgrâce ou une réprobation originelle quelconque. Par sa naissance (elle est née d'un mariage clandestin), par son origine (elle est fille de la pécheresse qu'il faut châtier), par son caractère (elle porte les signes d'une disposition tournée vers le malheur), Ériphile est une victime récusable. Enfin et surtout Ériphile ne meurt pas à la place d'Iphigénie par la voie qui était destinée à celle-ci. Dérobant le couteau du prêtre sacrificateur, elle l'enfonce en son sein, entendant mettre au compte des futurs agresseurs de sa patrie, un meurtre ».

On est loin de l'identification hérö̈que de l'Iphigénie d'Euripide au salut de la Grèce et de la célébration qui la rapproche des dieux :

« Tu m'as nourrie pour faire de moi une lumière de la Grèce,

Je n'ai pas honte de mourir $\gg$ (Euripide, 1502).

Et André Green de conclure : «En ce sens, l'idée de sacrifice est entièrement subvertie. Le sacrifice d'Ériphile ne coute rien à personne. Il est son destin et sa jouissance. Il n'apaise aucun dieu puisque personne ne s'est dépossédé d'elle dont aucun parent ne se soucie ».

La réécriture de Michel Azama est en apparence plus proche de la fable antique dans son dénouement: Iphigénie est sur l'autel. Kalkas tient le couteau sacrificiel indique l'avant- 
dernière didascalie (tableau 17:67). Mais le discours de la victime récuse explicitement l'identification héroïque pour dénoncer dans une antithèse l'inanité du sacrifice :

« Ne faites pas de moi

une statue enterrée par les siècles.

Faites de moi ce que je suis :

morte pour la guerre pour le plaisir des dieux.

Morte pour rien

et ma vie fut bien plus et bien moins qu'une vie. »

\section{Un sacrifice, des sacrifices}

Pour singulier que soit le sacrifice, sujet de la fable, il s'inscrit, comme acte rituel, dans la continuité d'autres qui l'ont précédé, chaque dépossession volontaire scellant l'alliance entre les dieux et les hommes. Chez Euripide, dès l'entrée sur scène du Chœur, le mot est employé au pluriel, renforcé par l'adjectif «innombrable», pour évoquer le lieu qui jouxte celui de l'action :

« Pour venir, j'ai traversé le bois des sacrifices innombrables d'Artémis » (v. 186)

Dans la bouche de l'Achille de Racine, le mot au pluriel désigne les rites autant pompeux que vains auxquels il oppose sa propre valeur au combat. Répliquant à Ulysse qui lui reproche de préférer l'hymen à la patrie, il s'insurge :

«Dans les champs phrygiens les effets feront foi

Qui la chérit le plus ou d'Ulysse ou de moi.

Jusque-là je vous laisse étaler votre zèle.

Vous pouvez à loisir faire des vœux pour elle.

Remplissez les autels d'offrandes et de sang.

Des victimes vous-même interrogez le flanc.

Du silence des vents demandez-leur la cause.» (I-2, v. 198)

Disqualifiés par le fils d'une déesse chez Racine, les sacrifices le sont chez Michel Azama par Apollon lui-même: il s'oppose ainsi aux autres dieux dans une parodie de débat démocratique :

« Le sang n'a-t-il pas assez coulé sur nos autels ?

Ne sommes-nous immortels que pour offrir la mort? » (Le jugement des dieux, p. 16)

Employé au singulier, le mot sacrifice peut recevoir chez les protagonistes une interprétation différente, source d'ironie tragique. À Iphigénie qui l'interroge, Agamemnon répond : « Il y a un sacrifice d'abord que j'ai à faire ici.» (Euripide, v. 673) et ses propos énigmatiques n'empêchent pas Iphigénie de demander « Nous allons donc danser, père, autour de l'autel ?» (v. 676). Quant à Clytemnestre, elle ne mesure pas l'abime qui sépare le rite propitiatoire précédant l'hymen de l'action à laquelle s'est résolu son époux : «As-tu fait à la déesse le sacrifice préliminaire, pour notre enfant ?» (v. 718)

Racine joue de la même équivoque dans la première scène où le père se trouve face à sa fille. Par une modalisation en discours second (dit-on), Iphigénie tente de percer une tristesse qui l'inquiète «Chalchas, dit-on prépare un pompeux sacrifice ? » (II-3, v. 574), « l'offrira-t-on bientôt ? » (v. 575), « Verra-t-on à l'autel votre heureuse famille ? » (v. 575)

L'ironie tragique culmine dans la pièce contemporaine par un procédé très voisin : Iphigénie interroge son père en se fondant sur une connaissance prétendument partagée, marquée par l'emploi du déterminant défini (la cérémonie, le sacrifice). 
«IPHIGENIE

Pourrai-je être présente à la cérémonie ?

AGAMEMNON

Les dieux exigent ta présence.

IPHIGENIE

Alors que ce jour soit béni.

Je ne savais pas que les dieux se préoccupaient autant de moi.

Je voulais voir le sacrifice

et ils m'ont exaucée.

AGAMEMNON

Tais-toi.

Quand les dieux veulent nous punir

ils exaucent nos prières. » (Dernière aube, Iphigénie à son père, p. 38 )

Mais c'est dans l'ouverture d'Iphigénie ou le péché des dieux que la détermination la plus subversive du nom sacrifice se rencontre :

«KRONOS

La puissante Artémis s'estimant offensée exige un sacrifice.

PLUSIEURS DIEUX

Oui oui un sacrifice un sacrifice !

Enfin.

On s'ennuie ici.

La vie d'immortel c'est mortel.

Un sacrifice un sacrifice. Vive Artémis ! (Le jugement des dieux, p. 11)

À la disproportion entre la réalité de l'offense (modalisée par le verbe s'estimer) et la rigueur de la punition s'ajoute l'indétermination totale: n'importe quel sacrifice, pourvu qu'il divertisse les dieux.

\section{Du pacte au sacrifice, ciment de l'alliance des Grecs contre Troie}

La fable peut être lue aussi comme un mythe fondateur de la nation grecque. Le sacrifice humain, qui doit ouvrir les portes de Troie, a été précédé par un premier pacte. Agamemnon le rappelle dans la première scène chez Euripide. Les rivalités étaient telles pour obtenir la main d'Hélène que son père Tyndare, pour sortir de l'impasse, eut l'idée de

«Faire jurer les prétendants, lier entre elles, Leurs mains droites, sur des victimes brûlées,

Verser des libations et prendre cet engagement solennel :

Lorsque l'un d'eux aurait la fille de Tyndare pour femme,

De se liguer tous pour la défendre si quelqu'un la lui prenait dans sa maison

Et s'en allait avec elle, en évinçant celui à qui appartenait le lit,

De faire la guerre et d'abattre la ville,

Par les armes, qu'elle fût grecque ou barbare, pareillement » (v. 58-65)

Hélène enlevée, Ménélas invoque l'ancien serment de Tyndare et Agamemnon prend la tête de l'armée. Il se trouve pris dans une autre impasse (le terme est repris quelques vers plus loin).

«Calchas, le devin, dans l'impasse où nous étions,

nous a répondu de sacrifier Iphigénie, née de ma semence,

À Artémis qui habite cette plaine

Et qu'alors il y aurait traversée et égorgement des Phrygiens, 
Si l'on sacrifie. Si l'on ne sacrifie pas, cela ne sera pas » (v. 88-93)

Les deux récits se font écho dans la bouche du même personnage, Agamemnon : impasse, sacrifice unissant les hommes d'une même nation contre les autres.

Chez Racine, le serment de Tyndare est repris mais avec des variantes majeures. Ulysse y est substitué à Ménélas. Mais alors que ce dernier, après avoir accusé son frère de trahison, lui recommande de ne pas tuer son enfant, l'Ulysse de Racine met Agamemnon en accusation en lui prêtant un rôle dévolu par la tradition à Ménélas :

« N'est-ce pas vous, Seigneur de qui la voix pressante

Nous a tous appelés aux campagnes du Xanthe,

Et qui de ville en ville, attestiez les serments

Que d'Hélène autrefois firent tous les amants,

Quand presque tous les Grecs, rivaux de votre frère,

La demandaient en foule à Tyndare votre père ?

De quelque heureux époux que l'on dût faire le choix

Nous jurâmes dès lors de défendre ses droits ;

Et si quelque insolent lui volait sa conquête,

Nos mains du ravisseur lui promirent la tête.

Mais sans vous ce serment que l'amour a dicté,

Libres de cet amour, l'aurions-nous respecté ? » (I, 3, v. 296-308)

L'oracle de Calchas se fait en présence d'Ulysse (I, 1, v. 53) qui met en balance la vie d'une innocente et la gloire du roi des rois, détournant lui aussi le sens du sacrifice :

«Le seul Agamemnon, refusant la victoire,

N'ose d'un peu de sang acheter tant de gloire ? » (I, 3, v. 317)

Quant à Achille, il n'est pas lié par le serment de Tyndare chez Racine, ni ne craint le pouvoir des dieux.

Dans la pièce contemporaine, toute allusion au serment de Tyndare a disparu. Seule subsiste l'attente des guerriers que chante la Coryphée :

« La gloire de tuer brûle leur cœur

la gloire de dépouiller des ennemis illustres

de répandre le sang noir des Troyens.

Mais le vent est mort les hommes s'énervent [...]

Mais la mer et les vents immobiles refusent à ces guerriers l'illustre hécatombe.

Oh les dieux!»

La course effrénée de l'Achille racinien vers une mort glorieuse semble devenue le modèle général, jusqu'à ce que l'Achille de la pièce contemporaine se révolte contre «le péché des dieux $\gg$ :

«Cette guerre que vous préparez je ne la ferai pas.

Non.

Je ne la ferai pas.

Je ne serai pas un héros grec.

Je suis « aristos achaïon », le meilleur des Grecs.

J'ai porté seul le poids de plusieurs batailles.

Non, ce n'est pas la peur qui parle par ma bouche.

Iphigénie

tu ne mourras pas. » (p.69)

\section{Sacrifier, un verbe trivalent}


Après ces différentes contextualisations, il est permis de revenir à la construction même du verbe sacrifier. Il s'agit d'un verbe à trois actants appelés par son sens et sa construction (Meleuc, 2000). Nous reprenons à Jacqueline Picoche (2002) la schématisation adoptée dans son Dictionnaire du français usuel : A1 sacrifie A2 à A3.

Nous montrerons que selon les réécritures, non seulement les valeurs prises par l'agent A1, le patient A2 et bénéficiaire A3 varient, mais que le verbe sacrifier lui-même entre en relation de parasynonymie avec des verbes trivalents (que nous avons laissé en caractères droits), ainsi que des verbes non trivalents (que nous avons mis en italiques pour souligner les différences sémantiques). S'agissant de tragédies, la mention du personnage (colonne de gauche, entre parenthèses en abrégé) est essentielle à l'interprétation.

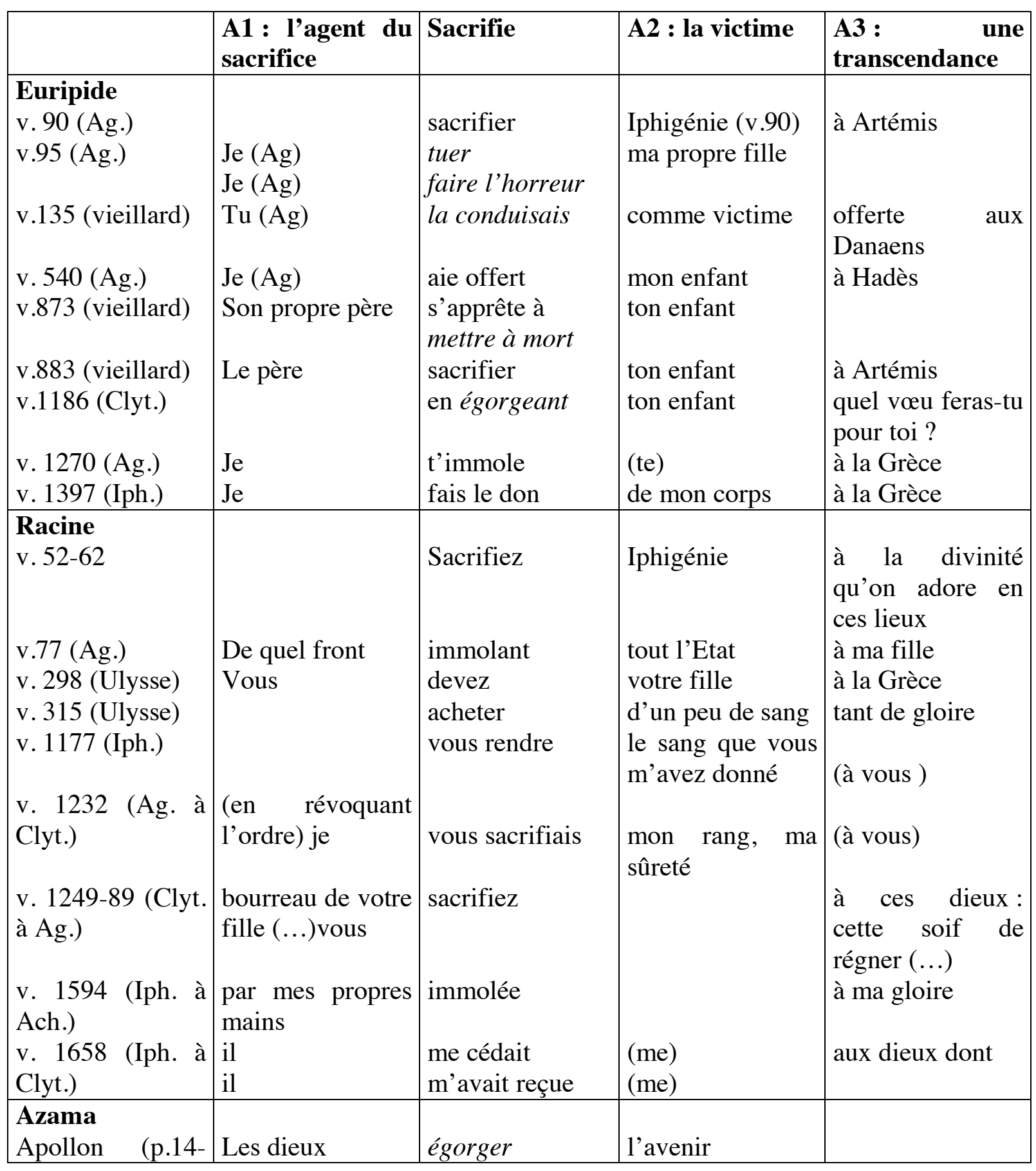




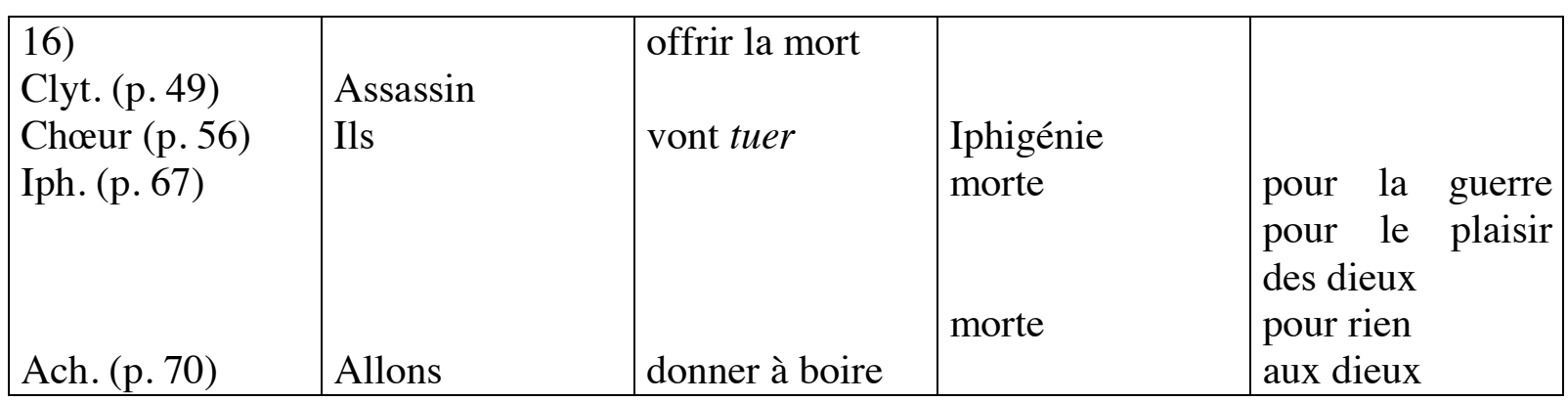

Dans la tragédie d'Euripide, l'horreur du meurtre (exprimée avec des verbes non trivalents : tuer, faire l'horreur) côtoie chez un même personnage la sublimation dans l'offrande ou le don (exprimée avec des verbes trivalents, euphémisant le sacrifice : offrir, faire le don), tandis que la dimension politique de la fable l'emporte au dénouement sur la signification religieuse du sacrifice.

Chez Racine, le mythe fondateur de la nation masque mal les enjeux de pouvoir : les verbes sacrifier et immoler sont mis en équivalence avec acheter par Ulysse : à la transcendance se substitue la gloire, et dans la bouche de Clytemnestre la soif de régner. Une autre série de verbes trivalents, donner, céder et recevoir, fondés sur la réciprocité d'un échange désintéressé, exaltent l'amour filial jusqu'à la dépossession. Le contraste entre ces deux séries est un puissant ressort du pathétique.

Les verbes non trivalents égorger, tuer et mourir saturent la pièce contemporaine. L'absurdité du but, dénoncée dès la première scène par Apollon, l'est ensuite par Clytemnestre, le Chœur, Iphigénie ainsi qu'Achille et la tragédie s'achève par la folie d'Achille, perversion du rite dionysiaque où le sang remplace le vin.

\section{Un double mouvement d'abstraction et d'expression de la subjectivité}

Selon les emplois, les actants d'un verbe trivalent peuvent être ou non exprimés. Employé de façon absolue, c'est-à-dire sans compléments, le verbe sacrifier est ouvert à plusieurs interprétations et permet parfois de maintenir l'équivoque. Il en est de même pour le nom sacrifice, selon qu'il est employé ou non sans compléments. C'est alors dans le contexte que des synonymes peuvent être recherchés. Rapportés au personnage qui les énonce, ils reçoivent des marques de subjectivité différentes, créant une polyphonie dans le pathétique. La dénonciation du meurtre reste dans un registre élevé, conforme au genre de la tragédie chez Euripide et Racine, tandis que dans la pièce contemporaine, la métaphore animale (boucherie, abattoir) ravale les victimes au rang d'animaux et se charge de connotations historiques aux deux conflits mondiaux du XXe (boucherie, abattoir, carnage) siècle et au franquisme (Vive la mort!).

\begin{tabular}{|l|l|l|l|}
\hline & $\begin{array}{l}\text { Verbe en } \\
\text { construction absolue } \\
\text { ou dépourvu d'un } \\
\text { actant }\end{array}$ & $\begin{array}{l}\text { Nom de procès avec } \\
\text { une interprétation } \\
\text { agentive }\end{array}$ & $\begin{array}{l}\text { Nom de procès avec } \\
\text { une interprétation } \\
\text { résultative }\end{array}$ \\
\hline Euripide & $\begin{array}{l}\text { Si l'on sacrifie } » \\
(\mathrm{v} .93)\end{array}$ & $\begin{array}{l}\text { Meurtre sanglant } \\
\text { (Ag., v. 510) } \\
\text { Sacrifice (Ag., v. 674) } \\
\text { Egorgement (Clyt., v. }\end{array}$ & $\begin{array}{l}\text { Mort (v. 658, 1382, } \\
1425)\end{array}$ \\
\hline
\end{tabular}




\begin{tabular}{|c|c|c|c|}
\hline & & \begin{tabular}{|l}
$906)$ \\
Un sacrifice humain \\
(Choeur, v. 1524) \\
L'offrande (Chalchas, \\
v. 1572)
\end{tabular} & \\
\hline Racine & $\begin{array}{l}\text { "vous sacrifiez à ces } \\
\text { dieux » (v. 1249-89, } \\
\text { Clyt. à Ag.) }\end{array}$ & $\begin{array}{l}\text { Supplice (Ag., 90) } \\
\text { La fureur de ce noir } \\
\text { sacrifice (Ag. 120) } \\
\text { Un meurtre } \\
\text { abominable (Clyt., v. } \\
921) \\
\text { Supplice (Clyt., v. } \\
938) \\
\text { Horrible festin (Clyt., } \\
\text { v. 1249) }\end{array}$ & $\begin{array}{l}\text { Métonymie : mon } \\
\text { sang (Iph., v. 1494) }\end{array}$ \\
\hline Azama & $\begin{array}{l}\text { Sacrifions, sacrifions. } \\
\text { Du sang! (Les dieux, } \\
\text { p. 11) }\end{array}$ & $\begin{array}{l}\text { Un sacrifice (Les } \\
\text { dieux, p. 11) } \\
\text { Ô ignoble boucherie } \\
\text { (Ag., p. } 43 \text { ) }\end{array}$ & $\begin{array}{l}\text { Une mort innocente } \\
\text { (Tisérias, p. 23) } \\
\text { Je ne serai plus rien } \\
\text { (Iph., p. 65) }\end{array}$ \\
\hline La folie d'Achille & $\begin{array}{l}\text { Tuons. Tuons. (Ach., } \\
\text { p. 70) }\end{array}$ & $\begin{array}{l}\text { Que ce crime fasse de } \\
\text { ta maison } \\
\text { L'ivresse. Le carnage. } \\
\text { L'hécatombe. }\end{array}$ & $\begin{array}{l}\text { un abattoir au sol } \\
\text { trempé de sang (Ach., } \\
\text { p. 70) } \\
\text { Vive la mort ! }\end{array}$ \\
\hline
\end{tabular}

\section{Du mot au discours : le sacrifice dans les sentences cachées}

Nommer le sacrifice, c'est déjà le justifier ou le condamner, comme on vient de le montrer. Dans chacune des pièces, ces choix énonciatifs s'accompagnent de choix discursifs : des sentences plus ou moins explicites y apparaissent. Elles ont en commun une relative autonomie syntaxique, énonciative et textuelle: ce sont des formes brèves, fonctionnant comme des topoï à valeur argumentative, servis par un jeu sur le signifiant (rythme, récurrences phoniques ou lexicales, figures). Toujours rapportées à un personnage, elles font entendre à travers lui une doxa qui entre en tension avec d'autres.

Comparer ces sentences permet de sensibiliser à l'écriture théâtrale et à la réception de ces formules par des publics de différentes époques. Chez Euripide, si Iphigénie accepte son sort, c'est comme fondateur d'une identité nationale marquée par des oppositions fortes (Grecs/ barbares ; esclaves/ gens libres):

«Il est plus important qu'un seul homme voie le jour plutôt que cent mille femmes. [...]

Il est normal que Grecs commandent aux barbares, mère, et non les barbares

Aux Grecs ; l'esclave est d'un côté, les autres sont des gens libres.» (v. 1394-1400)

Mais comme le remarque André Green (1967 : 195), la justification qui précède est difficile à entendre aujourd'hui : «L'économie d'Euripide concentre sur la seule Iphigénie cette marque de la cruauté divine et fait taire la révolte des Grecs, pourvoyeuse de morts. En outre, la 
suprématie masculine qui dicte le souci de préserver chaque vie de combattant interdit que l'on risque d'amoindrir ses forces avant la rencontre avec l'adversaire ».

Dans la pièce contemporaine, l'idéal héroïque est dénoncé par celui qui est censé l'incarner parfaitement dans un tableau nommé ironiquement nommé La belle mort :

LE TREMBLEUR

C'est la plus belle mort celle en plein vol de la jeunesse

ACHILLE

«Plutôt vivre en esclave qu'être roi chez les morts. (p. 60)

Quant à Iphigénie, c'est elle qui énonce avec force l'inanité du sacrifice dans un décasyllabe ponctué par les trois termes de négation :

« Aucun martyr jamais ne sert à rien.» (p. 67)

Ses dernières paroles font écho à la prophétie d'Apollon dans le premier tableau :

« Aucune pluie jamais ne lavera ce sang ».

Michel Azama a réintroduit le chœur à qui les dramaturges antiques confient la dimension réflexive du genre (Louvat, 1997) et prêtent la voix endeuillée de la tragédie contre le logos civique (Louraux, 1999). Racine lui, comme les dramaturges de son époque confie aux personnages eux-mêmes les discours sentencieux mais en estompe la valeur déontique pour les fondre dans le discours des personnages. Ainsi Iphigénie justifie la décision de son père jsuqu'à ses derniers mots, face à la révolte de Clytemnestre : "Il me cédait aux dieux, dont il m'avait reçue » (V3, v.1658)

Au terme de ce parcours, des étudiants et des élèves auront lu chacune des pièces pour ellemême et comme un développement possible de la signification de la fable. L'attention portée à une unité lexicale indissociable du sujet de ces pièces ne les conduit pas à des relevés écrasant la spécificité des écritures mais à des mises en relation, toujours rapportées à un niveau d'analyse explicite: lexique (construction des mots), sémantique référentielle (détermination dans le groupe nominal), sémantique lexicale (synonymes en contexte), syntaxe (constructions du verbe sacrifier et du nom correspondant), rhétorique (sentences).

Les étudiants, ayant mené la lecture tabulaire sur les trois œuvres intégrales (tâches étant réparties par groupes), ont gagné en autonomie dans le choix d'extraits pour une comparaison des emplois du mot sacrifice au lycée; ils sont en mesure de justifier le niveau d'analyse retenu. On peut faire l'hypothèse que ce jeu constant de décontextualisation/ recontextualisation du mot sacrifice contribue au développement de la conscience sémantique pour lequel les programmes de 2000 pour le lycée avait tracé trois directions: la contextualisation lexicale, textuelle et historique, en suggérant de nombreuses démarches. Il est regrettable que les programmes de lycée actuels s'en tiennent à un propos très général, citant seulement «la formation des mots et l'évolution de leurs significations ». Les programmes de lycée professionnel (2009) en revanche problématisent chaque objet d'étude et le déclinent en différents champs de savoirs (littérature, linguistique, histoire des arts) : le lexique n'y figure plus seulement à travers des notions abstraites ou des champs lexicaux mais à travers des mots dont la signification doit être travaillée pour poser la problématiques. C'est ce que nous avons tenté de faire pour Iphigénie.

Marie-Laure Elalouf

\footnotetext{
${ }^{3}$ On se reportera au travail sur le mot héros à propos du parcours de personnage : Adèle Blanc-Sec : http://www.lettres-histoire.ac-versailles.fr/spip.php?article781 et laux propositions de séances à partir du Dictionnaire du français usuel : http://lettreshg.ac-orleans-tours.fr/ressources_lettres/langue/
} 
Université de Cergy-Pontoise-école interne IUFM marie-laure.elalouf@iufm.u-cergy.fr

\section{Bibliographie}

\section{Oeuvres}

AZAMA, M. (1991). Iphigénie ou le péché des dieux. Paris : éditions Théâtrales. (11, 28 euros)

Iphigénie (Pocket n6138) : réunit la première tragédie d'Euripide et celle de Racine.

Critiques et références linguistiques ou didactiques

AHR, S. (2008). Iphigénie, un mythe tragiquement moderne (approche didactique).

http://www.versailles.iufm.fr/pdf/publi/documents/iphigenie_sylviane.pdf

BÉNICHOU, P. (1967). L'écrivain et ses travaux. Paris : J. Corti.

BONNEFOY, Y (dir.) (1981). Dictionnaire des mythologies. Paris : Flammarion.

ESCOLA, M. (1998). Présentation de l'Iphigénie de Racine dans la collection GF Flammarion.

GREEN, A. (1969). Un xil en trop, le complexe d'Edipe dans la tragédie, Éditions de minuit. LOUVAT,B. (1997). Poétique de la tragédie classique. Paris, SEDES.

LORAUX, N. (1999). La Voix endeuillée. Essai sur la tragédie grecque. Paris : Gallimard, "Les Essais ".

Meleuc, S. (2000). " Pour un traitement lexical du verbe », Le français aujourd'hui $\mathrm{n}^{\circ} 131$, construire les compétences lexicales. Paris, Armand Colin

PiCOCHE, J. \& Rolland, J.-C. (2002). Dictionnaire du français usuel. Bruxelles, De Boeck.

REY-DEBOVE, J. (2004). Le Robert Brio. Paris, éditions Le Robert.

\section{Manuels}

ABENS, C. (dir.) (2011). Passeur de textes, livre unique 1.. Paris : WebLettres \& Le Robert DAUMAS, X. (dir.) (2011). Terres littéraires. Paris : Hatier.

ETERSTEIN, C. (dir.) (2011). Méthodes et pratiques, lycée. Paris : Hatier.

LABOURET, D ; (dir.) (2011). 2e, 1e, Méthodes et pratiques. Paris : Bordas.

SIVAN, P. (dir.) (2011). Français, méthodes, lycée. Paris : Nathan 